\title{
AICLE en acción: interacciones formativas y profesionales entre profesorado de todos los estamentos educativos
}

\section{CLIL in Action: Educational and Professional Interactions among Teachers}

M. ㄹ Elena Gómez Parra ${ }^{1}$, Cristina A. Huertas Abril², Ángela M. Larrea Espinar³, Antonio R. Raigón Rodríguez, Mercedes Osuna Rodríguez, David Bullejos Martín, Francisco Villamandos de la Torre, José Zamora Salido, Adela González Fernández, Cristina Gámez Fernández, Ana M. Calatayud Tenas, Leonor Martínez Serrano, Trinidad Jerez Montoya, Margarita Tejederas Dorado, Beatriz Martínez Serrano, Soledad Serrano Ariza, Elisa Pérez Gracia, José Manuel Almodóvar Antequera, Cristina Merino García, Emilio Arjona Crespo, Francisco J. Palacios Hidalgo, Angélica Hilinger Alegre, Cristina Díaz Martín

Fecha de recepción: 17/10/2018; Fecha de revisión: 06/03/2019; Fecha de aceptación: 21/05/2019

Cómo citar este artículo:

Gómez Parra, M.를. Huertas Abril, C. A., Larrea Espinar, A. M., Raigón Rodríguez, A. R., Osuna Rodríguez, M., Bullejos Martín, D., Villamandos de la Torre, F., Zamora Salido, J., González Fernández, A., Gámez Fernández, C. M. $\stackrel{a}{\text {, }}$ Calatayud Tenas, A. M. ${ }^{a}$, Martínez Serrano, L. M. ${ }^{a}$, Jerez Montoya, T., Tejederas Dorado, M., Martínez Serrano, B., Serrano Ariza, S., Pérez Gracia, E., Almodóvar Antequera, J. M., Merino García, C., Arjona Crespo, E., Palacios Hidalgo, F. J., Hilinger Alegre, A., y Díaz Martín, C. (2019). AICLE en acción: interacciones formativas y profesionales entre el profesorado en formación, el profesorado en ejercicio, el profesorado universitario y los CEP de Córdoba y Provincia. Revista de Innovación y Buenas Prácticas Docentes, 8(2), 78-89.

\footnotetext{
I Universidad de Córdoba (España), elena.gomez@uco.es; CÓDIGO ORCID: https://orcid.org/0000-00017870-3505.

2 Universidad de Córdoba (España), cristina.huertas@uco.es; CÓDIGO ORCID: https://orcid.org/00000002-9057-5224.

3 Universidad de Córdoba (España), ff1laesa@uco.es; CÓDIGO ORCID: https://orcid.org/0000-0001-60499905.
} 


\title{
Resumen:
}

AICLE (Aprendizaje Integrado de Contenidos y Lenguas Extranjeras) es el enfoque oficial europeo para la educación bilingüe, auspiciado por la Comisión Europea y fomentado por una cantidad ingente de investigación e implementación en todos los niveles educativos. Sin embargo, AICLE necesita de más investigación en el aula e interacciones que ofrezcan datos que nos indiquen en qué contextos y bajo qué condiciones específicas las acciones educativas son o no eficaces. Es en esta línea (innovación educativa e interacción en el aula) en la que entendemos que la formación inicial del profesorado en el ámbito de la educación bilingüe ha de estar necesariamente ligada a la formación permanente del profesorado y a la praxis en el aula que proporciona el profesorado en ejercicio.

El objetivo esencial de este artículo consiste en establecer el vínculo entre profesorado universitario, profesorado en ejercicio, profesorado en formación (a tres niveles: Grado, Máster y Doctorado) y los CEP de Córdoba y provincia para diseñar una estructura de formación recíproca y cuidadosamente planificada, que contribuya a mejorar la calidad de la educación bilingüe a través del intercambio de prácticas de innovación educativa que fomenten la interacción y el aprendizaje en acción.

Palabras clave: AICLE, interacciones formativas, interacciones profesionales, educación bilingüe.

\begin{abstract}
:
CLIL (Content and Foreign Language Integrated Learning) is the official European approach to bilingual education, sponsored by the European Commission and fostered by an enormous amount of research and implementation at all levels of education. However, CLIL needs more research in the classroom and interactions which provide data on what contexts and under what specific conditions educational actions are or are not effective. It is in this line (educational innovation and interaction in the classroom) that we understand that the initial training of teachers in the field of bilingual education must necessarily be linked to the ongoing training of teachers and to the praxis in the classroom provided by practising teachers.

The essential objective of this article is to establish the link between university teachers, practising teachers, teachers in training (at three levels: Bachelor, Master and Doctorate) and the CEPs of Cordoba and province to design a carefully planned reciprocal training structure that contributes to improving the quality of bilingual education through the exchange of educational innovation practices that foster interaction and learning in action.
\end{abstract}

Key Words: CLIL, educational interactions, professional interactions, bilingual education. 


\section{INTRODUCCIÓN}

El Aprendizaje Integrado de Contenidos y Lenguas Extranjeras (AICLE) es el enfoque oficial a nivel europeo para la educación bilingüe desde la educación infantil (como se recoge en Language Learning at Preprimary Level: Making It Efficient and Sustainable: A Policy Handbook, Comisión Europea, 2011) hasta la enseñanza permanente de adultos (como se establece en la resolución Multilingualism: an Asset for Europe and a Shared Commitment, Comisión Europea, 2014). En la última década, España ha sido testigo de un aumento considerable de programas AICLE en los niveles de enseñanza primaria y secundaria (Madrid \& Hughes, 2011; Pérez-Cañado, 2012; Martínez, 2012; Marsh, Pérez-Cañado \& Ráez, 2015), que se está extendiendo al sector universitario (Ramos-García, 2013; Madrid \& Madrid, 2014; Madrid \& Julius, 2017). Sin embargo, AICLE necesita de más investigación en el aula e interacciones que ofrezcan datos que nos indiquen en qué contextos y bajo qué condiciones específicas estas acciones son o no eficaces y, por tanto, sirven a las medidas para las que fueron inicialmente pensadas y diseñadas.

Desde una perspectiva abarcadora, el Plan Estratégico de Desarrollo de las Lenguas en Andalucía (PEDLA) define AICLE como el "Enfoque metodológico mediante el cual una materia o parte de ella se enseña a través de una lengua extranjera con un objetivo doble: el aprendizaje de contenidos de determinadas materias curriculares y el aprendizaje simultáneo de una lengua extranjera" (Consejería de Educación - Junta de Andalucía, 2016, p. 51). El PEDLA, que supone un nuevo compromiso con el plurilingüismo en Andalucía, pone de relieve que en la evaluación del Plan de Fomento del Plurilingüismo se establecieron vías de mejora en la formación del profesorado, elemento clave tanto en su fase inicial universitaria como en la formación permanente (PEDLA, 2016, p. 9). Así, el PEDLA destaca que "no es posible un sistema educativo de calidad y equidad sin un profesorado motivado y con una formación adecuada" (Consejería de Educación - Junta de Andalucía, 2016, p. 22).

Es en esta línea (que aúna calidad en la docencia, equidad, motivación del profesorado, formación adecuada e innovación pedagógica) en la que entendemos que la formación inicial del profesorado en el ámbito de la educación bilingüe ha de estar necesariamente ligada, por un lado, a la formación permanente del profesorado y, por otro, a la praxis en el aula que proporcionan los docentes en ejercicio. El programa institucional de proyectos de innovación educativa de la Universidad de Córdoba ha financiado un proyecto enmarcado en la modalidad 4 de dicha convocatoria, (titulada Proyectos que impulsen la colaboración con centros docentes públicos no Universitarios), así como en las Instrucciones de 14 de febrero de 2017 de la Dirección General de Innovación, para la participación de los centros docentes públicos no Universitarios en intervenciones en el ámbito de la innovación y la investigación educativa realizadas en colaboración con las universidades andaluzas. Este proyecto se marcó el objetivo fundamental de establecer un puente esencial en educación bilingüe (con enfoque AICLE) entre la formación inicial y permanente del profesorado. Esto se debe, por una parte, a que el profesorado en formación de los Grados en Educación Infantil y Primaria, tanto en sus itinerarios bilingües como en el caso de la Mención en Lengua Extranjera, así como el alumnado de los Másteres en Educación o Formación de Profesorado, no suele disponer de interacciones directas con docentes en activo fuera de sus períodos de Practicum, que se limitan a un número concreto de horas por curso y a un Centro de Educación Infantil y Primaria (CEIP) o Instituto de Enseñanza Secundaria (IES) determinado; y, por otra, a la importancia de la formación permanente que se establece desde la Delegación de Educación de Córdoba a través de los CEP en el seno de la articulación de su Plan de Plurilingüismo. 
Tras la fundamentación teórica de este trabajo, describiremos la propuesta de innovación educativa que sustenta este proyecto de innovación educativa. A continuación, ofreceremos nuestra reflexión sobre los resultados obtenidos, que revelará la mejora que ha supuesto este proyecto para la formación inicial del profesorado. Finalmente, discutiremos la posible repercusión que esta innovación pedagógica puede reportar para las futuras generaciones de profesorado de educación bilingüe.

\title{
2. FUNDAMENTACIÓN TEÓRICA
}

El contexto educativo en el que nos vemos inmerso se encuentra claramente definido por una sociedad europea donde el plurilingüismo y la multiculturalidad son dos de sus características clave. En él, el Aprendizaje Integrado de Contenidos y Lenguas Extranjeras (AICLE), también conocido por sus siglas en inglés CLIL (Content and Language Integrated Learning), es el enfoque fundamental de la educación bilingüe en el contexto europeo. AICLE no solo fomenta el aprendizaje de lenguas extranjeras, sino que "El alumnado ha de ser capaz de construir el contenido mediante la mejora de sus competencias lingüísticas, así como de desarrollar habilidades de aprendizaje y estrategias que le permitan aprender mediante esa segunda lengua de manera solvente" (Gómez, 2017a, p. 197). En este sentido, AICLE es un modelo ambicioso, holístico y de grandes expectativas, cuya implementación está siendo acompañada de una cantidad ingente de investigación e innovación educativa que analizan aspectos claves como la ratio del alumnado, el nivel tanto del profesorado como del alumnado en segundas lenguas (Huertas, 2016), la influencia de las TIC (Huertas, 2017), el uso de materiales didácticos o la influencia de las familias en el sistema. Sin embargo, y como señala Gómez (2017b), ha de tenerse en cuenta que:

\begin{abstract}
...a pesar del éxito que los programas bilingües tienen a nivel mundial, aún se necesita una cantidad razonable de trabajo y de investigación en este tema, ya que cuestiones cruciales sobre diferentes aspectos de su implementación, sobre los métodos y enfoques más apropiados de enseñanza y aprendizaje, y sobre sus interrelaciones con la educación intercultural surgen aún en forma de grandes interrogantes entre el profesorado, investigadores y alumnado (p. 9).
\end{abstract}

Para alcanzar el desarrollo adecuado de estas competencias en el alumnado, el papel del profesorado es fundamental en tanto que ha de emplear una metodología que permita la integración de las denominadas 4C del enfoque: contenidos, cognición, cultura y comunicación (Coyle, Hood \& Marsh, 2010). Por todo ello, la formación del profesorado ha de ocupar un lugar destacado, pues son numerosos los estudios que señalan la existencia de carencias, especialmente en la formación inicial (Wolff, 2005; Lorenzo, Casal, Moore \& Alfonso, 2009; Mellado, Bolarín \& Porto, 2013; Travé, 2013; Pérez-Cañado, 2016, entre otros). En esta misma línea, Coonan (2011) afirma que el enfoque AICLE ha hecho al profesorado consciente de que ha de recibir formación específica para el desarrollo de su profesión. De hecho, Lasagabaster (2008) destaca que, de acuerdo con los datos obtenidos en su investigación, el nivel en segundas lenguas del profesorado es bajo, tanto para el profesorado de contenidos, como para el profesorado de lenguas (dato que, por otra parte, resulta preocupante en Andalucía siguiendo el estudio de Lorenzo, Casal \& Moore, 2009). 
A tenor de todo lo expuesto anteriormente, podemos afirmar que la formación específica del profesorado (inicial y permanente) en el enfoque AICLE se torna un tema fundamental para la mejora de los resultados educativos en el contexto de la educación bilingüe.

\section{OBJETIVOS}

El objetivo principal de este proyecto de innovación educativa es, pues, establecer el vínculo necesario (que viene ya demandado desde hace tiempo en el campo de la educación bilingüe) entre profesorado universitario, profesorado en ejercicio, profesorado en formación (a tres niveles: Grado, Máster y Doctorado) y los CEP de Córdoba y provincia para diseñar una estructura de formación recíproca y cuidadosamente planificada, que contribuya a mejorar la calidad de la educación bilingüe a través del intercambio de prácticas de innovación educativa que fomenten la interacción y el aprendizaje en acción entre todos los niveles de la formación del profesorado.

Con el fin de alcanzar el objetivo principal, se establecieron los siguientes objetivos secundarios:

- Establecer puntos de encuentro, tanto de carácter formativo como profesional, entre profesorado en ejercicio de Educación Primaria y Secundaria y profesorado en formación.

- Establecer puntos de encuentro, tanto de carácter formativo como profesional, entre profesorado en ejercicio de Educación Primaria y Secundaria y profesorado universitario, en ambos casos tanto pertenecientes a Áreas Lingüísticas $(\mathrm{AL})$ como a Áreas No Lingüísticas (ANL).

- Difundir las experiencias derivadas de buenas prácticas en Educación Primaria y Secundaria que se están llevando a cabo en distintos centros educativos de Córdoba y provincia.

- Facilitar la formación permanente de profesorado en ejercicio de Educación Primaria y Secundaria gracias a metodologías de innovación pedagógica en AICLE basadas en mindfulness y design thinking.

- Reflexionar sobre la necesidad de la formación continua en el ámbito de la educación bilingüe, especialmente en el enfoque AICLE.

\section{MATERIAL Y MÉTODOS}

El presente proyecto de innovación y mejora de la praxis educativa y de la calidad universitaria se erige como la primera fase de una serie estructurada de intervenciones, basadas en el uso de la enseñanza situada, que se desarrollarán mediante seminarios participativos e interactivos con cuatro colectivos implicados: profesorado universitario de la UCO, profesorado en formación (alumnado de Grado, Máster y Doctorado de la UCO), Centros del Profesorado de la Delegación Territorial de Educación de Córdoba y profesorado en ejercicio (principalmente de Educación Primaria y Educación Secundaria). 


\subsection{Participantes}

Este proyecto de innovación sobre praxis educativa, enmarcado dentro del proyecto "AICLE en acción", involucra a los siguientes colectivos dentro de la comunidad educativa universitaria y no universitaria:

- Docentes en formación que cursan sus estudios en la Universidad de Córdoba, concretamente de dos niveles educativos:

- Alumnado de 4ํㅡㄴ curso del Grado de Educación Primaria (Itinerario Bilingüe y Mención Lengua Extranjera: inglés).

- Alumnado del Máster en Estudios Ingleses Avanzados (Lingüística Cognitiva/Literatura) y Educación Bilingüe.

- Docentes de Educación Primaria en ejercicio, que imparten su docencia en centros bilingües que siguen el enfoque AICLE, pertenecientes a CEIP que destacan por sus buenas prácticas docentes y que fueron seleccionados por los tres Centros del Profesorado (CEP) de la Delegación Territorial de Educación de Córdoba: CEP Luisa Revuelta de Córdoba, CEP Priego-Montilla y CEP Sierra de Córdoba. Concretamente, en el curso 2017/2018 se trató de profesorado perteneciente a los siguientes centros: CEIP Al-Yussana (Lucena, Córdoba), CEIP Alcalde Pedro Barbudo (Córdoba) y CEIP Cronista Rey Díaz (Córdoba).

- Personal de Delegación Territorial de Educación de Córdoba, liderado por las Responsables de Plurilingüismo, quien a través de los tres CEP de la Delegación de Córdoba seleccionaron los CEIP implicados en este proyecto de innovación docente y participaron activamente en la impartición de algunos seminarios.

- Profesorado universitario tanto de Áreas Lingüísticas $(A L)$ y Áreas No Lingüísticas (ANL) con experiencia en proyectos de innovación docente previos sobre metodología AICLE, quien ejerció la coordinación global del proyecto.

\section{RESULTADOS}

\subsection{Resultados obtenidos de las interacciones formativas desde el profesorado en ejercicio al profesorado en formación}

Las interacciones formativas en educación bilingüe desde el profesorado en ejercicio al profesorado en formación constaron de una serie de seminarios con el objetivo fundamental de acercar la realidad del aula a los futuros docentes, y hacerlos conscientes de los puntos fuertes y débiles que la praxis diaria del profesorado en ejercicio encuentra en la implementación del enfoque AICLE en sus aulas.

Al ser la primera fase del proyecto, los seminarios se desarrollaron durante los meses de diciembre de 2017 y enero de 2018, de modo que el profesorado en formación ya tuviera el conocimiento esencial que le permitiese aprovechar esta acción de 
innovación docente, al tiempo que pudiera entender en su totalidad los conceptos, la metodología y los recursos que el profesorado en ejercicio emplea en sus aulas AICLE. Asimismo, el profesorado en formación, en este momento del curso académico, ya había diseñado una Unidad Didáctica Integrada (UDI) bajo la dirección del profesorado participante en este proyecto, pues había cursado las asignaturas correspondientes al 4ํㅜㄴ curso Grado de Educación Primaria (Itinerario Bilingüe y Mención Lengua Extranjera: Inglés) o del Máster en Estudios Ingleses Avanzados (Lingüística Cognitiva/Literatura) y Educación Bilingüe, ambos de la UCO. Se ha de señalar que, a estas sesiones, a su vez, asistió parte del profesorado universitario participante con el objetivo de analizar y evaluar la sesión, a fin de extraer datos que han servido de feedback y mejora del proyecto en años sucesivos.

Se organizó un total de cinco seminarios (véase figura 1) donde se abordaron, de manera progresiva y gradual, aspectos fundamentales del enfoque AICLE y sus implicaciones en el aula en contextos reales de CEIP de Córdoba y provincia.

\begin{tabular}{|c|c|c|}
\hline № seminario & Temática & $\begin{array}{l}\text { Profesorado } \\
\text { implicado }\end{array}$ \\
\hline 1 & $\begin{array}{l}\text { La educación bilingüe y plurilingüe en Andalucía: } \\
\text { conceptos clave de AICLE, implementación del } \\
\text { plurilingüismo en contextos educativos reales y } \\
\text { evolución del Plan de Fomento del Plurilingüismo } \\
\text { (PFP) al Plan Estratégico de Desarrollo de las } \\
\text { Lenguas en Andalucía (PEDLA) }\end{array}$ & $\begin{array}{l}\text { Responsable de } \\
\text { Plurilingüismo de la } \\
\text { Delegación Territorial } \\
\text { de Educación de } \\
\text { Córdoba }\end{array}$ \\
\hline 2 & $\begin{array}{l}\text { Aspectos clave para llevar a cabo la Dirección de } \\
\text { un centro bilingüe, cómo se implantó el programa } \\
\text { bilingüe en un centro concreto (CEIP Al-Yussana, } \\
\text { Lucena), cómo se coordinan las áreas lingüísticas } \\
\text { (AL) y las áreas no lingüísticas (ANL) }\end{array}$ & $\begin{array}{l}\text { CEIP Al-Yussana } \\
\text { (Lucena, Córdoba) }\end{array}$ \\
\hline 3 & $\begin{array}{l}\text { Cómo se desarrolla el enfoque AICLE teniendo en } \\
\text { cuenta la atención a la diversidad }\end{array}$ & $\begin{array}{l}\text { CEIP Alcalde Pedro } \\
\text { Barbudo (Córdoba) }\end{array}$ \\
\hline 4 & $\begin{array}{l}\text { El proyecto Cantalenguas: creación musical en } \\
\text { lenguas extranjeras gracias a la coordinación de } \\
\text { áreas lingüísticas (AL) y áreas no lingüísticas } \\
\text { (ANL) }\end{array}$ & $\begin{array}{l}\text { CEIP Cronista Rey } \\
\text { Díaz (Córdoba) }\end{array}$ \\
\hline 5 & $\begin{array}{l}\text { Nuevos enfoques metodológicos: el uso de la } \\
\text { biodanza en la asignatura de Educación Física } \\
\text { bilingüe }\end{array}$ & $\begin{array}{l}\text { CEP - Delegación } \\
\text { Territorial de } \\
\text { Educación de Córdoba }\end{array}$ \\
\hline
\end{tabular}

Tal y como se recoge en la figura 1, para la celebración de los distintos seminarios se siguió un proceso discursivo descendente, lo cual permitió una progresión de lo más general a lo más específico. Para ello, a su vez, siempre se tuvo en cuenta la importancia del aprendizaje significativo gracias a la difusión de buenas praxis docentes auténticas, desarrolladas en Centros de Educación Infantil y Primaria de Córdoba y provincia. 


\title{
5.2 Resultados obtenidos de las interacciones formativas desde el profesorado en ejercicio al profesorado en formación
}

Las interacciones formativas en educación bilingüe desde el profesorado universitario al profesorado en ejercicio de Educación Primaria y Educación Secundaria constaron de dos seminarios basados en metodologías de innovación pedagógica en AICLE, concretamente en mindfulness y design thinking, lo que previamente se acordó con el personal de la Delegación de Córdoba y los CEP. Estos seminarios se programaron, como avanzábamos, de manera que, por un lado, la especialización del profesorado AICLE estuviese lógicamente adecuada a las necesidades identificadas previamente por la Delegación de Educación y los CEP de Córdoba y, por otro, siguiesen una línea lógica de especialización del profesorado, que se ha constituido en una forma diferente de innovación docente en AICLE.

En esta segunda fase del proyecto, los seminarios se desarrollaron durante los meses de enero y febrero de 2018. Por una parte, la teoría de mindfulness (junto con sus aplicaciones educativas) es una temática reciente que implica una cantidad ingente de investigación e innovación educativa por los buenos resultados que ofrece. Mindfulness es un término inglés, con origen en pali, que hace referencia a conciencia, atención y recuerdo (Siegel, Germer \& Olendzki, 2009). Germer (2005) señala las aplicaciones de mindfulness en tres áreas: i) como constructo teórico; ii) como práctica (habitualmente prácticas de meditación); y iii) como proceso psicológico (es decir, el estado de consciencia). En el ámbito educativo, mindfulness se aplica en diversas áreas y en casi todos los niveles (desde educación infantil hasta educación superior) y su finalidad es, de acuerdo con Simón (2016) "cambiar la mente para ver con claridad" ( $p$. 28). Las aplicaciones de mindfulness en el aula son numerosas, y diversas investigaciones destacan sus beneficios. Como indica Reber (2014):

\begin{abstract}
I reviewed the core studies on mindful learning and noticed that much research not labeled as studies on mindfulness provided insights that can plausibly be explained by mindfulness theory. We saw that cognitive load hampers learning if it does not integrate different perspectives; but that it promotes learning when it varies perspectives on the same theme; that invention activities as a mindful activity increase subsequent learning; that interest can be increased by interventions that take principles of mindful learning into account; and that students may benefit from principles of mindfulness in order to deal with adverse feelings, for example when watching debates that are styled as confrontational (p. 1066).
\end{abstract}

Por otra parte, design thinking es una metodología que busca resolver problemas de manera sistemática a fin de reducir los riesgos y aumentar sus posibilidades de éxito. Brown (2008) define design thinking como:

A discipline that uses the designer's sensibility and methods to match people's needs with what is technologically feasible and what a viable business strategy can convert into customer value and market opportunity. [...] It often entails a great deal of perspiration (p. 86).

Aunque se originó en el mundo empresarial, design thinking pasó fácilmente al ámbito educativo (Kimbell, 2011), y su praxis conlleva la implementación de un proceso de aprendizaje participativo consistente en cinco pasos: 
1. Empatizar: es un aprendizaje sobre la audiencia, mediante observación y entrevistas.

2. Definir: busca la creación de un punto de vista de acuerdo con las necesidades y las perspectivas del usuario.

3. Idear: esta fase se establece mediante un proceso de brainstorming o tormenta de ideas que busca aportar posibles soluciones al problema planteado.

4. Prototipar: en este momento del proceso, se construye una representación del problema, de manera que pueda mostrarse a otros.

5. Testar: finalmente, el usuario comprueba y testa la idea prototipada, con la finalidad de conseguir retroalimentación.

Como se puede comprobar, se trata de un proceso en el que la participación y la colaboración del grupo se torna esencial para la consecución de los objetivos. Asimismo, sea fomenta el aprendizaje desde un punto de vista creativo y supone, en sus diferentes aplicaciones, un cambio revolucionario desde una perspectiva innovadora.

La aplicación de ambas metodologías innovadoras en el ámbito de la educación bilingüe, y más concretamente en el enfoque AICLE, tuvo muy buena acogida entre el profesorado en ejercicio de Educación Primaria y Secundaria. Se ha de señalar que a estos seminarios también asistió parte del profesorado universitario participante en el proyecto con el objetivo de analizar y evaluar la sesión, y poder extraer datos que sirvan de feedback y mejora del proyecto en años sucesivos.

\section{CONCLUSIONES}

El desarrollo y la implementación de este proyecto de innovación docente nos ha permitido adelantar interesantes resultados relativos al nivel de implementación del enfoque AICLE en Córdoba y provincia, lo cual esperamos poder continuar en cursos posteriores. La reciente finalización del proyecto no nos ha permitido analizar con detalle todos los datos, pero sí contamos con las declaraciones de todos los participantes, que nos permiten afirmar que este proyecto está suponiendo una verdadera innovación educativa debido a la fructífera interacción entre distintos colectivos de profesorado (pertenecientes a la formación inicial y a la formación permanente), además de la sinergia que supone el aprendizaje de la teoría y de la buena praxis en las aulas bilingües.

Por una parte, y sin menoscabo de los resultados finales que el análisis de los datos indique, entendemos que el profesorado en ejercicio ha encontrado en este proyecto un constructo fundamental para acercarse a la formación inicial del profesorado desde una perspectiva de compañeros en la formación. Por otra, las interacciones formativas en educación bilingüe desde el profesorado en ejercicio al profesorado en formación han dado lugar a una "práctica reflexiva" en el contexto docente en un doble sentido. Así, el profesorado en ejercicio ha de reflexionar acerca de cómo desarrolla su docencia para poder explicarla y transmitirla al profesorado en formación; mientras que el profesorado en formación puede reflexionar sobre la práctica real en el aula, con la que aún no se ha enfrentado completamente. De igual modo, entendemos que para el profesorado universitario este proyecto es una magnífica oportunidad para comprobar cómo se lleva a la praxis del aula los fundamentos teóricos del enfoque AICLE, al tiempo que puede observar in situ cómo el alumnado, futuros docentes, pone en marcha lo aprendido.

Por todo lo anterior, consideramos que este proyecto no debe considerarse solo como una innovación docente, sino como un conjunto de estrategias efectivas, significativas y motivadoras para todos los colectivos implicados, en tanto que todos ellos pueden participar activamente y beneficiarse de las buenas prácticas docentes de 
los otros, contribuyendo así a una mejora tanto en la formación como en el ejercicio profesional del profesorado bilingüe en distintos niveles educativos.

Creemos, por tanto, que esta es una línea de trabajo en la que debemos insistir, ya que la formación del profesorado inicial y permanente puede beneficiarse de la sinergia positiva proveniente del grupo de trabajo diseñado ex profeso, y consideramos de gran interés la continuación de "AICLE en acción" en años sucesivos, partiendo de seminarios de especialización y actualización en AICLE recorriendo los distintos cursos y asignaturas que se imparten en los centros bilingües de Educación Primaria.

\section{AGRADECIMIENTOS}

Este trabajo ha sido posible gracias al Plan de Innovación y Buenas Prácticas Docentes 2017-2018 de la Universidad de Córdoba, concretamente en su Modalidad 4 "Proyectos que impulsen la colaboración con centros docentes públicos no universitarios".

\section{REFERENCIAS}

Brown, J., Collins, A. \& Duguid, P. (1989). Situated cognition and the culture of learning. Educational Researcher, 18(1), 32-42.

Brown, T. (2008). Design Thinking. Harvard Business Review, 84-92.

Comisión Europea. (2011). European Strategic Framework for Education and Training (ET 2020). Language Learning at Pre-primary School Level: Making it Efficient and Sustainable. A Policy Handbook. Recuperado de https://goo.gl/kmAQiY

Comisión Europea. (2014). Multilingualism - an asset and a commitment. Recuperado de: https://goo.gl/HvoN1x

Consejería de Educación - Junta de Andalucía. (2016). Plan Estratégico de Desarrollo de las Lenguas en Andalucía. Horizonte 2020. Recuperado de: https://www.juntadeandalucia.es/export/drupaljda/plan estrategico.pdf

Coonan, C. M. (2011). CLIL in teacher training. Studi di Glottodidattica, 2, 1-14.

Coyle, D., Hood, P. \& Marsh, D. (2010). Content and Language Integrated Learning, Cambridge: Cambridge University Press.

Derry, S., Levin, J., y Schauble, L. (1995). Stimulating statistical thinking through situated simulations. Teaching of Psychology, 22(1), 51-57.

Díaz, F. (2003). Cognición situada y estrategias para el aprendizaje significativo. Revista electrónica de investigación educativa, 5(2), 1-13.

Eurydice. (2006). CLIL at School in Europe. Bruselas: Comisión Europea. Recuperado de http://www.indire.it/lucabas/lkmw file/eurydice/CLIL EN.pdf

Germer, C. K. (2005). Mindfulness: What Is It? What Does It Matter? En C. K. Germer, R. D. Siegel, y P. R. Fulton (Eds.), Mindfulness and psychotherapy (pp. 3-27). Nueva York, NY, US: Guilford Press.

Gómez, M. ${ }^{a}$ E. (2017a). Evaluación de resultados de la educación bilingüe. En M.a I. Amor-Almedina, R. Serrano-Rodríguez \& E. Pérez-Gracia (Coords.), La Educación Bilingüe desde una visión integrada e integradora (pp. 193-202). Madrid: Síntesis. 
Gómez, M. E. (2017b). Prefacio. En M. a E. Gómez-Parra, y R. Johnstone (Eds.), Educación Bilingüe: Tendencias Educativas y Conceptos Clave / Bilingual Education: Educational Trends and Key Concepts (pp. 9-10). Madrid: Ministerio de Educación, Ciencia y Cultura.

Hendricks, C. (2001). Teaching causal reasoning through cognitive apprenticeship: What are results from situated learning? The Journal of Educational Research, 94(5), 302-311.

Huertas, C. A. (2016). La competencia oral en lengua inglesa de los futuros maestros: propuesta metodológica y estrategias de comunicación. En M. a I. AmorAlmedina, J.L. Luengo-Almena, \& M. Martínez-Atienza (Eds.), Educación intercultural: metodología de aprendizaje en contextos bilingües (pp. 113-120). Granada: Atrio.

Huertas, C. A. (2017). The Role of Technology in the Development of Materials for Bilingual Education. En M. aㅡ E. Gómez-Parra \& R. Johnstone (Eds.), Educación Bilingüe: Tendencias Educativas y Conceptos Clave / Bilingual Education: Educational Trends and Key Concepts (pp. 209-220). Madrid: Ministerio de Educación, Ciencia y Cultura.

Kimbell, L. (2011). Rethinking Design Thinking: Part I. Design and Culture, 3(3), 285306.

Lasagabaster, D. (2008). Foreign Language Competence in Content and Language Integrated Courses. The Open Applied Linguistics Journal, 1(1), 30-41.

Lorenzo, F., Casal, S. \& Moore, P. (2009). The effects of Content and Language Integrated Learning in European education: Key findings from the Andalusian bilingual sections evaluation project. Applied Linguistics, 1(3), 418-442.

Lorenzo, F., Casal, S., Moore, P. \& Alfonso, Y. M. (2009). Bilingüismo y Educación: Situación de la Red de Centros Bilingües en Andalucía. Sevilla: Fundación Centro de Estudios Andaluces.

Madrid, D. \& Hughes, S. (Eds.), (2011). Studies in bilingual education. Berna: Peter Lang.

Madrid, D., \& Julius, S. M. (2017). Quality Factors in Bilingual Education at the University Level. Porta Linguarum, 28, 49-66.

Madrid, M. \& Madrid, D. (2014). La formación inicial del profesorado para la educación bilingüe, Granada: Editorial Universidad de Granada.

Marsh, D., Pérez-Cañado, M. L. \& Ráez, J. (Eds.). (2015). CLIL in action: voices from the classroom. Newcastle-upon-Tyne: Cambridge Scholars Publishing.

Martínez, J. de D. (2012). Teaching and learning English through bilingual education. Newcastle-upon-Tyne: Cambridge Scholars Publishing.

Mellado, M. L., Bolarín, M. J. \& Porto, M. (2013). Programas bilingües en Educación Primaria: valoraciones de docentes. Porta Linguarum: Revista Internacional de Didáctica de las Lenguas Extranjeras, 20, 253-268.

Pérez-Cañado, M. ${ }^{a}$ L. (2012). CLIL research in Europe: past, present, and future", International Journal of Bilingual Education and Bilingualism, 15(3), 315-341.

Pérez-Cañado, M. ${ }^{a}$ L. (2016). Are teachers ready for CLIL? Evidence from a European study. European Journal of Teacher Education, 39(2), 202-221.

Ramos, A. M. (2013). Higher education bilingual programmes in Spain. Porta Linguarum, $19,101-111$.

Reber, R. (2014). Mindfulness in Education. En A. Le, C.T. Ngnoumen \& E. J. Langer (Eds.), The Wiley Blackwell Handbook of Mindfulness (pp. 1054-1070). Chichester: Blackwell.

Siegel, R. D., Germer, C. K. \& Olendzki, A. (2009). Mindfulness: What is it? Where did it Come from? En F. Didonna (Ed.), Clinical Handbook of Mindfulness, (pp. 17-35). New York: Springer.

Simón, V. (2016). Aprender a practicar mindfulness. Barcelona: Sello Editorial.

Travé, G. (2013). Un estudio sobre las representaciones del profesorado de Educación Primaria acerca de la enseñanza bilingüe. Revista de Educación, 361, 379-402. 
Wolff, D. (2005). Approaching CLIL. Project D3 - CLIL Matrix. The CLIL quality matrix, Central Workshop Report 6/2005, 10-25. 